\title{
On cancer-fighting nanorobots
}

\section{Editorial}

Nanorobotics refers to the design and building of nano-sized devices (or nanorobots) in the size range $\left(10^{2}-10^{4}\right.$ nanometers) constructed of nanoscale or molecular components. For brain applications (glioblastomas), sizes of interest are less than 100 nanometers. Such devices can move with nanoscale precision. While still in research and development, these nanomachines may find important applications in medicine, especially in cancer treatment where they can be used to identify and destroy cancer cells all the while sparing healthy cells. Because of their small size, nanorobots would need to be in very large numbers and to work fast together to perform microscopic and macroscopic tasks. These nanorobot swarms could theoretically come in two flavors, those that replicate themselves without any constraints in the natural environment and those that do not replicate. In the fight against cancer, only the latter are currently considered and utilized.

There have recently been two major developments and advances in this field, namely ${ }^{1}$ the construction of an autonomous DNA nanorobot as an intelligent drug delivery and therapeutic system that responds to molecular triggers in vivo $a^{2} d^{2}$ the successful programming of autonomous nanorobots to shrink tumors by cutting-off their blood supply (an antiangiogenic approach, see Fymat). ${ }^{3}$ The structures are built from DNA that can self-fold in all sorts of shapes and sizes.

In the former instance, using a DNA origami (or Trojan horse) approach (see, e.g., Fymat), ${ }^{4-8}$ the nanorobot was programmed to transport therapeutic drugs and deliver them at the tumor site. ${ }^{9}$ The nanorobot is a two-layer structure with the blood coagulation protease thrombin within its inner cavity and an exterior shell functionalized with a DNA aptamer. An aptamer usually consists of a short strand of oligonucleotides or peptide molecules that bind to a specific target molecule. It serves two roles, to bind nucleolin and subsequently act as a molecular trigger for mechanically opening the nanorobot and releasing its thrombin payload. Nucleolin is a protein specifically expressed in large amounts on the surface of tumor-associated endothelial cells but not found on the surface of healthy cells. The thrombin is specifically delivered at tumor-associated blood vessels to induce intravascular thrombosis, provoking tumor necrosis and inhibiting tumor growth. In mice and Bima miniature pigs, the researchers showed that the nanorobot is safe and immunologically inert with no detectable changes in normal blood coagulation or cell morphology. Most importantly, there was no evidence of the nanorobots spreading into the brain where they could cause unwanted side effects, such as a stroke. After attacking tumors, most of the nanorobots were cleared and degraded from the body after 24 hours. By two days, there was evidence of advanced thrombosis and, after three days, thrombi in all tumor vessels were observed. The technology is a promising strategy for precise drug delivery in cancer therapy. ${ }^{10}$

In the second instance, a nanorobot was successfully programmed to shrink tumors by inducing blood coagulation that cuts-off the blood supply. The approach was shown to have high therapeutic efficacy and safety profiles in multiple solid tumors using DNA-based nanocarriers. Each nanorobot is made from a flat, rectangular DNA
Volume 9 Issue 5 - 2018

\author{
Alain L Fymat \\ International Institute of Medicine and Science, California, USA
}

Correspondence: Alain L Fymat, International Institute of Medicine and Science, California, USA, Tel (760) 507-6862, Email alain.fymat@fiimas.org

Received: October 19, 2018 | Published: October 23, 2018

origami sheet, 90X60 nanometers in size. This appears to be the first (or one of the very first) fully autonomous DNA robotic cancer seekand-destroy-by-starvation system. It was demonstrated in rodents utilizing various cancer types (breast, skin, ovaries, lung). By blocking the tumor blood flow and clotting the blood vessels that feed the tumor growth, thrombin causes a sort of "tumor mini-heart attack", leading to tumor tissue death. In a melanoma mouse model, the nanorobot not only affected the primary tumor but also prevented the formation of metastasis, showing a promising therapeutic potential. Since all solid tumor-feeding blood vessels are essentially the same, the approach can be utilized for many types of cancers. It may help accomplish the ultimate goal of cancer research, namely the eradication of solid tumors and vascularized metastases.

\section{References}

1. Fymat AL. Recent Research Developments in Anti-Cancer Therapy. $J$ Cancer Prev Curr Res. (2016a);5(2):1-2.

2. Fymat AL. Nanotechnology and Cancer. J Cancer Prev Curr Res. (2016b);5(6):1-7.

3. Fymat AL. Antiangiogenic Targeting of Early Developing Glioblastoma Behind a Weakened Blood Brain Barrier. J Tumor Med Prev. (2017c);2(3):1-6

4. Fymat AL. Recent Developments in Nanomedicine Research. J Nanomed Res. (2016c);4(4):1-12.

5. Fymat AL. Nanochemotherapy: An Emergent Anti-Cancer Modality. Glob J Nano. (2017a);1(1):1-6.

6. Fymat AL. Nanooncology: Perspective on Promising Anti-Tumor Therapies. J Tumor Med Prev. (2017b);1(1):1-10.

7. Fymat AL. Nanobiotechnology Advances in Oncology. Adv Bioeng Biomed Sci Res. (2018a);1(1):1-6.

8. Fymat AL. Roles of Nanomedicine in Clinical Neurocience. Glob $J$ Nanomed. (2018b);4(1):1-3.

9. Li S, Jiang Q, Liu S, et al. Cancer-fighting nanorobots programmed to seek and destroy tumors. Nanowerks News. 12 February.

10. Yan H Zhao Y, Ding B, Nie G, et al. A DNA nanorobot functions as a cancer therapeutic in response to a molecular trigger in vivo. Nature Biotechnology. 2018. 